\title{
Rapid Quantification of Urinary Organic Acids by HPLC Mass Spectrometry to Assess Nutritional Individuality in Chinese Children
}

\author{
Qichen Zhang, ${ }^{*}$ Jia You,** Wenli ZHU,*** Zhigang WU,***广 and Jingyuan XıONG**† \\ *Chengdu Women's and Children's Central Hospital, School of Medicine, University of Electronic Science \\ and Technology of China, Chengdu, 611731, China \\ **West China School of Public Health and Healthy Food Evaluation Research Center, Sichuan University, \\ Chengdu, 610041, China \\ ***Chengdu MediMass Technology CO., LTD, Chengdu, 611135, China
}

\begin{abstract}
A urinary organic acids profile can be utilized as an effective screening tool for analyzing abnormality of nutrient metabolism. By using these metabolic markers in conjunction with one another, it helps in understanding how individual nutrient metabolism is executed and to determine where there may be imbalances in the metabolic cycle. In this study, we developed a rapid quantification method of 20 urinary organic acids by HPLC-mass spectrometry. A pre-analytical process of organic acid extraction from a urine sample is crucial in this methodology. The process was accomplished by liquid-liquid extraction followed by strong anion exchange. Compared with previous methods, this method greatly reduces the analysis time and allows for the simultaneous quantification of 20 organic acids within 10 min for the first time. This methodology enabled us to analyze urine samples collected from 34 Chinese children. The abnormalities of some urinary organic acids were found in this group, which revealed evidence of functional inadequacy of specific nutrients. The preliminary data in this study confirmed the suitability of the method for rapid and accurate quantification of the target organic acids in urine samples.
\end{abstract}

Keywords Nutrient metabolism, urinary organic acids, mass spectrometry, nutritional individuality, Krebs cycle

(Received February 5, 2020; Accepted May 1, 2020; Advance Publication Released Online by J-STAGE May 8, 2020)

\section{Introduction}

Organic acids are a broad class of compounds involved in fundamental metabolic processes. Derived from dietary proteins, fats and carbohydrates, they are used by the body to generate cellular energy for cell functions. ${ }^{1}$ Organic acid profiles were traditionally used for the early detection or the monitoring of inherited disorders of amino acid and organic acid metabolism, which is so-called "Inborn Errors of Metabolism" $($ IEOM $) .^{2}$ Nowadays organic acid testing can also serve as important diagnostic indicators of abnormal metabolism, such as energy metabolism and nutrient deficiencies. ${ }^{3-5}$ The selected urinary organic acids can be measured as important diagnostic indicators of abnormal metabolism, such as cellular energy production by the mitochondria or carbohydrate metabolism or B-complex deficiency and etc. ${ }^{5}$ For example, the citric acid cycle, or so-called Krebs cycle, occurs in the matrix of the mitochondrion, which uses a series of enzyme-catalyzed chemical reactions to generate usable energy for cellular supply. ${ }^{4}$ Blocks in the Krebs cycle may lead to muscle pain, chronic fatigue, and premature ageing. The impaired metabolism

Q. Z. and J. Y. contributed equally to this work.

† To whom correspondence should be addressed.

E-mail: wubox@yahoo.com (Z.W.); bear831006@qq.com (J. X.) indicates nutrient insufficiencies, which may be affecting a patient's health. Common symptoms may include: anemia, fatigue, elevated homocysteine, ischemic heart disease, stroke, deep vein thrombosis and etc. ${ }^{1,2,6-8}$ The organic acids testing measures certain metabolites to determine what blockages may be occurring in the metabolic pathways due to nutrient insufficiencies. As food is metabolized in the body, essential cofactors, such as the B group vitamins for the energy pathways of body cells, are required. ${ }^{9}$ If these cofactors are not fully provided, there are imbalances in the metabolic cycle, which result in elevated levels of certain urinary organic acids. ${ }^{6}$ Therefore, organic acid testing is capable of understanding how nutrient metabolism is executed and determining where there may be imbalances in the metabolic cycle. ${ }^{9-11}$

In this regard, it requires rapid and reliable methodologies for the analysis of large batches of urine samples, which is essential in clinical and epidemiological studies. Common methods for the quantification of urinary organic acids have been based on ion chromatography (IC), capillary electrophoresis (CE) and gas chromatography coupled to mass spectrometry (GC-MS). ${ }^{13-16}$ However, CE and IC lack robustness for the routine analysis of biological samples. On the other hand, although GC-MS can achieve good separation, it requires a complicated sample derivatization step to increase the sensitivities. It is a timeconsuming multi-step procedure possibly yielding different reaction rates, resulting in poor repeatability. Therefore, 
high-performance liquid chromatography coupled with mass spectrometry (HPLC-MS) became the best option for a robust high-throughput method for the rapid quantification of urinary organic acids. ${ }^{10,11,17,18}$

Because of the large amount and number of organic compounds presented in urine, it is really difficult to accurately quantify all of the organic acids of interest. Ideal isolation or extraction methods are required for high recoveries and good detection sensitivities. The most commonly used urine extraction procedure for organic acids involves solvent partition (liquidliquid extraction) or solid-phase extraction (SPE). Whereas according to the property of organic acids, strong anion exchange is also an option for sample extraction. Previously studies using strong anion exchange and some other disposable columns for the separation of organic acids in urine were reported. ${ }^{19,20}$ The precision, accuracy of extraction process for liquid-liquid extraction and solid phase extraction was compared. And the extraction efficiencies for important urinary metabolites were also investigated. ${ }^{21,22}$

The purpose of this work was to develop a reliable sample preparation method with good sensitivities for a rapid quantification of 20 urinary organic acids, and to use this methodology to study nutritional individuality in Chinese children.

\section{Experimental}

\section{Reagents and chemicals}

Twenty urinary organic acids include adipate, suberate, ethylmalonate, pyruvate, L-lactate, $\beta$-hydroxybutyrate, citrate, cis-aconitate, isocitrate, $\alpha$-ketoglutarate, succinate, fumarate, malate, hydroxymethylglutarate, $\alpha$-ketoisovalerate, $\alpha$ ketoisocaproate, $\alpha$-keto- $\beta$-methylvalerate, xanthurenate, $\beta$ hydroxyisovalerate and methylmalonate. Internal standards include D3-methylmalonate, D3-ethylmalonate and D4-citrate. All organic acids and internal standards have been reported to have chemical purity $>98 \%$, and were purchased from SigmaAldrich. Acetone, methanol, sodium bicarbonate, tri-fluoroacetic acid (TFA) and ethyl acetate were purchased from SigmaAldrich (St. Louis, USA). Purified water was obtained using a Milli-Q Plus ultrapure water system (Millipore, USA).

\section{Urine sample collection}

After $2 \mathrm{~mL}$ first-morning urine samples were collected from children (aged from $4-11$ ), the samples were stored at $-80^{\circ} \mathrm{C}$ prior to analysis immediately after collection. Urine samples were taken after the usual dietetic limitation (tea, coffee, chocolate, coke and etc.). None of the children received medications that could interfere with organic acids. The protocol was approved by the Ethical Committee of the Institute of Public Health and Healthy Food Evaluation Research Center Informed and written consent with the use of biological materials for research reasons was obtained from all parents of the subjects participating into the project.

\section{Sample preparation}

Stock solutions of 20 organic acids were each prepared at $80 \mu \mathrm{g} / \mathrm{mL}$ by dissolving $2 \mathrm{mg}$ of the powders in methanol to a final volume of $25 \mathrm{~mL}$ in a volumetric flask and stored at $-20^{\circ} \mathrm{C}$. At the beginning of each analysis, samples of the stock solutions were analyzed to verify that standards gave the same results as when they were freshly prepared. Charcoal stripped human urine that contains $0.1 \%$ L-ascorbic acid without detectable levels of the 20 organic acids was employed for the preparation of calibration standards.

\section{Liquid-liquid extraction}

After $2 \mathrm{~mL}$ of ethyl acetate was added to the urine sample equivalent to $1 \mu \mathrm{mol}$ of creatinine in a $10-\mathrm{mL}$ test tube with internal standard added, the mixture was supplemented with an aqueous hydroxylamine hydrochloride solution. The $\mathrm{pH}$ of the mixture was adjusted to 14 with $\mathrm{NaOH}$ and incubated for $30 \mathrm{~min}$ at $60^{\circ} \mathrm{C}$. After extraction, the solution was evaporated to dryness with nitrogen and then dissolved in methanol before analysis.

\section{Strong anion exchange}

A silica-based strong anion exchange (SAX) sorbent (Isolute, Mid Glamorgan, UK) was used in solid-phase cartridges (Thermo, Waltham, USA) for extraction. The SAX sorbent was first treated with methanol in $100 \mathrm{mM}$ acetic acid before application. After preconditioning and sample loading, urinary organic acids were eluted with methanol in $2 \%$ of formic acid. And the extracts were then evaporated to dryness and re-dissolved in methanol before analysis.

\section{HPLC-MS}

HPLC mass spectrometry analysis was performed using a LCMS-8050 Triple Quadrupole Liquid Chromatography Mass Spectrometry system (Shimadzu). Both the HPLC and mass spectrometer were controlled by LabSolutions software (Shimadzu). HPLC was carried out on an XSelect HSS T3 column $(3.5 \mu \mathrm{m}, 2.1 \times 100 \mathrm{~mm}$, Waters $)$ maintained at $45^{\circ} \mathrm{C}$. A total of $10 \mu \mathrm{L}$ of each sample was injected onto the column for separation. The mobile phase, operation at a flow rate of $0.3 \mathrm{~mL} / \mathrm{min}$, consisted of $0.2 \%$ trimethylamine $(\mathrm{v} / \mathrm{v})$ in water as solvent $\mathrm{A}$ and methanol as solvent B. A linear gradient, changing the $\mathrm{A} / \mathrm{B}$ solvent ratio from $18: 82$ to $10: 90$ in $10 \mathrm{~min}$, was employed. The general mass spectrometry conditions were as follows: ESI negative mode; spray voltage, $-4 \mathrm{kV}$; sheath and auxiliary gas, nitrogen; scan type, multiple reaction monitoring (MRM) mode; collision gas, argon. Precursor and fragment ions are: adipate $(\mathrm{m} / \mathrm{z}, 145.2-83.1)$, suberate $(\mathrm{m} / \mathrm{z}, 173.2$ 111.2), ethylmalonate $(\mathrm{m} / \mathrm{z}, 131.1-87.1)$, pyruvate $(\mathrm{m} / \mathrm{z}, 89.1$ 43.1), L-lactate $(\mathrm{m} / \mathrm{z} \quad 87.1-43.1), \quad \beta$-hydroxybutyrate $(\mathrm{m} / \mathrm{z}$ 103.1 - 59.1), citrate $(\mathrm{m} / \mathrm{z} 191.1-87.1)$, cis-aconitate $(\mathrm{m} / \mathrm{z}$ 173.1 - 85.1), isocitrate $(\mathrm{m} / \mathrm{z}, 191.1-73.1), \alpha$-ketoglutarate $(\mathrm{m} / \mathrm{z}$ 145.1 - 101.1), succinate $(\mathrm{m} / \mathrm{z} 117.1-73.0)$, fumarate $(\mathrm{m} / \mathrm{z}$ $115.1-71.0)$, malate $(\mathrm{m} / \mathrm{z} 133.1-115.0)$, hydroxymethylglutarate, $\alpha$-ketoisovalerate $(\mathrm{m} / \mathrm{z} 115.1$ - 115.1), $\alpha$-ketoisocaproate $(\mathrm{m} / \mathrm{z}, 129.2$ - 129.2), $\alpha$-keto- $\beta$-methylvalerate $(\mathrm{m} / \mathrm{z} 129.2$ - 129.2), xanthurenate $(\mathrm{m} / \mathrm{z} \quad 204.2-160.1), \beta$-hydroxyisovalerate $(\mathrm{m} / \mathrm{z}$ 117.1 - 73.1), methylmalonate $(\mathrm{m} / \mathrm{z} 117.1-73.1)$, D3methylmalonate $(\mathrm{m} / \mathrm{z} \quad 120.1-73.1)$, D3-ethylmalonate $(\mathrm{m} / \mathrm{z}$ 134.1 - 87.1) and D4-citrate $(\mathrm{m} / \mathrm{z} 195.1$ - 87.1).

\section{Range and linearity}

The linearity of the method was determined by analyzing calibration curves containing seven points with different nonzero concentrations. Organic acid standards were prepared by subsequent dilution of the standard solutions in water. Calibration curves were prepared on the day of analysis and analyzed with the QC samples and urinary samples as a single batch. The lowest concentration on the calibration curve was regarded as the limit of quantification (LOQ), with a signal-tonoise ratio $(S / N)$ of at least 10 and acceptable accuracy (within $\pm 20 \%$ of the nominal value) and precision (coefficient of variation $(\mathrm{CV})$ less than 20\%). The limit of detection (LOD) was determined as the concentration of the standard solution leading to a $S / N$ of least 3 . 

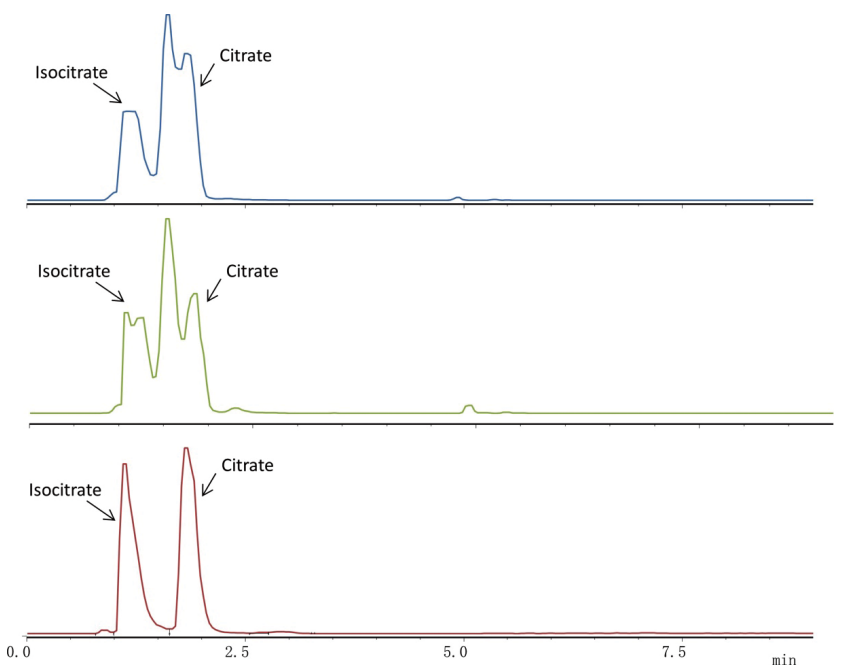

Fig. 1 Selected ion currency of citrate and isocitrate in urinary sample by HPLC mass spectrometry. Up, liquid-liquid extraction middle, strong anion exchange; bottom, liquid-liquid extraction, followed by strong anion exchange.

\section{Precision and accuracy}

Five replicates of QC samples at three different concentration levels (low, medium and high) were applied to calculate the precision and accuracy of the method. The intra-assay precision involved all organic acids per each concentration level performed within a single run. To examine the inter-assay precision, samples were prepared and analyzed five times in three different runs. The accuracy of the method was calculated with spiked samples.

\section{Results and Discussion}

The panel of organic acids selected in this approach are wellknown important diagnostic indicators of abnormal metabolism, which were used in most functional medicine labs world-wide for clinical applications. For example, adipate, suberate, and ethylmalonate are all functional markers for the deficiency of carnitine and vitamin B2. When insufficient levels of carnitine or vitamin B2 slow down the energy conversion in the mitochondria, other parts of the cellular machinery take over and make adipate and suberate. A similar block in another pathway causes high ethylmalonate. The 20 organic acids selected as well as internal standards formed deprotonated molecular ions in negative mode electrospray, which were chosen as parent ions in the MRM mode. Optimization of the MRM conditions was carried out by postcolumn infusion of standard solutions. Since isomeric or isobaric metabolites have the same $\mathrm{m} / \mathrm{z}$ values for both the precursor and product ions, the MRM alone could not differentiate between these metabolites. The chromatographic conditions were optimized for a complete separation of all of the target organic acids. All organic acid standards were chromatographic separated from isomeric and isobaric interferences, and each of them was confirmed by comparisons of the retention time, the precursor ion, product ions and MRM ratio with the respective values. However, because of the large number of organic acids and other metabolites presented in the urine matrix, it is extremely difficult to accurately quantify the target organic acids in a short analysis time without sample derivatization. Interference from other

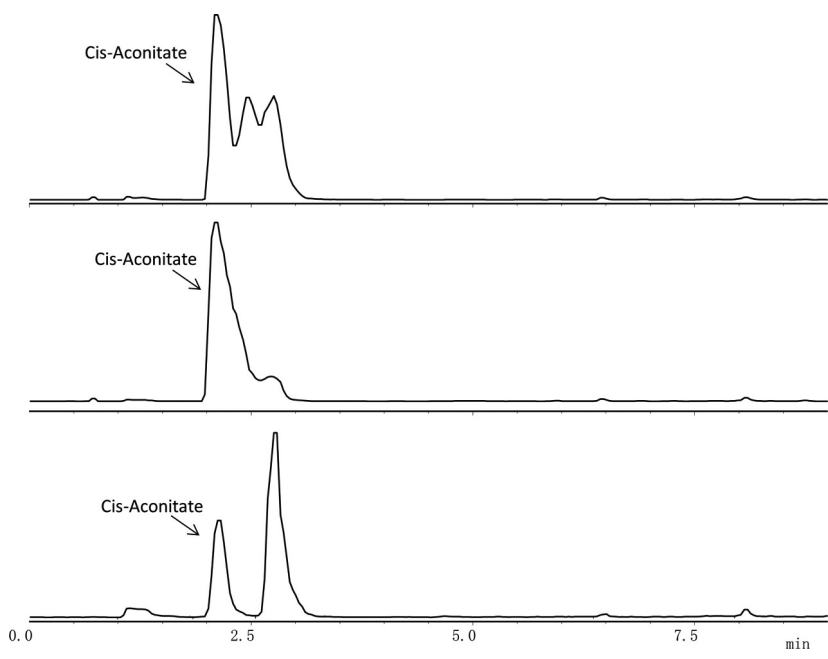

Fig. 2 Selected ion currency of cis-aconitate in urinary sample by HPLC mass spectrometry. Up, liquid-liquid extraction; middle, strong anion exchange; bottom, liquid-liquid extraction followed by strong anion exchange.

metabolites in the urine matrix has to be removed prior to chromatographic analysis, which could affect the target quantification dramatically. Therefore, extraction or purification methods must be applied to decrease matrix interference and to increase the detection sensitivities for the organic acids of interest. We compared liquid-liquid extraction and SPE with a strong anion-exchange sorbent for organic acids purification in urine samples. A comparison of organic acid quantification was presented with these two extraction procedures. Figure 1 shows MRM chromatograms of the same urine sample of citrate and isocitrate quantification. These two organic acids are isomeric metabolites and the MRM alone could not differentiate between these two. Therefore, baseline chromatographic separation is required for successful quantification of these isomeric metabolites. The top one in Fig. 1 was obtained after a liquidliquid extraction procedure, the middle one was obtained after a strong anion exchange procedure and the bottom one was obtained upon liquid-liquid extraction, followed by strong anion exchange. There was a lot of interference in the quantification of citrate and isocitrate by MRM after either liquid-liquid extraction or a strong anion-exchange pretreatment alone. Other urinary metabolites were present in high quantities that overlapped with, or masked, the two target peaks. Liquid-liquid extraction or strong anion exchange alone was not able to completely remove all those metabolites causing interference. Whereas in the sample that was treated with liquid-liquid extraction followed by strong anion exchange, the interference in the urine matrix was completely removed and the two isomers were baseline separated for accurate quantification (Fig. 1 bottom). Figure 2 shows MRM chromatograms of the same urine sample of cis-aconitate quantification. Similarly, liquidliquid extraction or strong anion exchange alone could not remove all the interference in urine either. High quantities of urinary metabolites overlapped with the target peak. When the urine sample was treated with liquid-liquid extraction followed by strong anion exchange, the interference peaks were removed and cis-aconitate was accurately quantified (Fig. 2 bottom). In samples treated with liquid-liquid extraction or strong anion exchange alone, there were always an overlapping of the target peaks due to incomplete removal of the urinary interference. The urinary sample must be treated with liquid-liquid extraction, 
followed by strong anion exchange to completely remove all interfering metabolites. After sample treatments, the 20 target organic acids were purified and rapid quantification was achieved by HPLC mass spectrometry. Compared with previous work on organic acids analysis, the present methodology allows for the rapid quantification of 20 targets within $10 \mathrm{~min}$. Table 1 shows the limit of detection, range and accuracy for each organic acid. Compared with results reported in literatures using different separation methods, range and accuracy of our approach were comparable. Some techniques have better sensitivity compared with our approach. For example, capillary electrophoresis, ion chromatography coupled with mass spectrometry could reach a good sensitivity in the nano-molar range. ${ }^{23}$ However, these methods are time-consuming and reported in 13 to $75 \mathrm{~min}$ cycle times. ${ }^{24,25}$ In regards of urinary organic acid profiles, the limit of detection, range and accuracy of our approach were good for routine laboratory practice.

The quantification of urinary organic acids is used in modern nutritional medicine as a simple and sensitive test to demonstrate the functional inadequacy of specific nutrients. The increase of certain organic acids in urine can provide useful information for an early diagnosis of metabolic disorders and nutritional problems. To study the nutritional individuality of Chinese children, quantification of the 20 target organic acids in urine samples collected from 34 children was performed using this
Table 1 The limit of detection, range and accuracy for each organic acid measured with HPLC-mass spectrometry

\begin{tabular}{llccc}
\hline No. & \multicolumn{1}{c}{ Target } & $\begin{array}{c}\text { Range/ } \\
\mu \mathrm{g} \mathrm{mL} \mathrm{m}^{-1}\end{array}$ & $\begin{array}{c}\text { LOD/ } \\
\mu \mathrm{g} \mathrm{mL} \mathrm{mL}^{-1}\end{array}$ & $\begin{array}{c}\text { Accuary, } \\
\%\end{array}$ \\
\hline 1 & Adipate & $20-0.5$ & 0.1 & $88-118$ \\
2 & Suberate & $20-0.5$ & 0.1 & $83-111$ \\
3 & Ethylamlonate & $20-0.5$ & 0.2 & $83-113$ \\
4 & Pyruvate & $40-1$ & 0.2 & $95-114$ \\
5 & L-Lactate & $100-2.5$ & 0.5 & $95-107$ \\
6 & $\beta$-Hydroxybutyrate & $20-0.5$ & 0.1 & $95-105$ \\
7 & Citrate & $1000-2.5$ & 2 & $82-112$ \\
8 & cis-Aconitate & $300-7.5$ & 1 & $83-118$ \\
9 & Isocitrate & $300-7.5$ & 1 & $85-102$ \\
10 & $\alpha$-Ketoglutarate & $100-2.5$ & 0.5 & $84-101$ \\
11 & Succinate & $50-1.25$ & 0.2 & $90-117$ \\
12 & Furnarate & $50-1.25$ & 0.2 & $92-104$ \\
13 & Malate & $50-1.25$ & 0.2 & $83-119$ \\
14 & Hydroxymethylglutarate & $40-1$ & 0.2 & $85-110$ \\
15 & $\alpha$-Ketoisovalerate & $5-0.125$ & 0.05 & $87-107$ \\
16 & $\alpha$-Ketoisocaproate & $5-0.125$ & 0.05 & $86-103$ \\
17 & $\alpha$-keto- $\beta$-Methylvalerate & $5-0.125$ & 0.05 & $90-118$ \\
18 & Xanthurenate & $5-0.125$ & 0.05 & $89-102$ \\
19 & $\beta$-Hydroxyisovalerate & $50-1.25$ & 0.2 & $93-109$ \\
20 & Methtylmalonate & $10-0.25$ & 0.05 & $92-112$ \\
\hline
\end{tabular}

Table 2 Concentrations of 20 target organic acids in 34 children aged from $4-11$ years old (The values higher than $95 \%$ reference range were in grey)

No. Name Age Sex Creatinine/ Adipate Suberate Ethylamlonate Pyruvate L-Lactate $\beta$-Hydroxybutyrate Citrate cis-Aconitate $\mathrm{mg} \mathrm{mL}^{-1}$

Mean $(n=3), \mu \mathrm{g} \mathrm{mg}^{-1}$ creatinine

\begin{tabular}{|c|c|c|c|c|c|c|c|c|c|c|c|c|}
\hline 1 & ZJX & 6 & M & 2.07 & 0.95 & 1.66 & 1.49 & 1.58 & 7.35 & 0.18 & 108.76 & 130.38 \\
\hline 2 & CZX & 5 & $\mathrm{~F}$ & 0.84 & 2.76 & 1.84 & 2.21 & 0.01 & 4.25 & 0.12 & 279.69 & 52.96 \\
\hline 3 & CCL & 6 & $\mathrm{M}$ & 1.89 & 3.70 & 2.01 & 2.82 & 0.23 & 7.61 & 0.90 & 301.66 & 105.16 \\
\hline 4 & HAQ & 6 & $\mathrm{~F}$ & 1.08 & 1.68 & 0.50 & 4.95 & BL & 2.81 & 0.24 & 110.43 & 58.77 \\
\hline 5 & FJY & 5 & $\mathrm{~F}$ & 0.68 & 1.25 & 1.13 & 1.28 & BL & 2.72 & 0.66 & 185.30 & 58.98 \\
\hline 6 & ZYZ & 7 & M & 0.57 & 0.82 & 1.67 & 0.76 & BL & 1.95 & 0.17 & 122.10 & 34.73 \\
\hline 7 & HXY & 8 & $\mathrm{~F}$ & 0.50 & 0.75 & 8.71 & 1.08 & 0.48 & 4.13 & 0.25 & 154.26 & 39.51 \\
\hline 8 & PJX & 10 & $\mathrm{M}$ & 1.68 & 3.73 & 2.83 & 2.04 & 1.79 & 1.81 & 0.85 & 172.35 & 123.56 \\
\hline 9 & FLC & 11 & M & 1.02 & 2.89 & 2.64 & 2.18 & 0.27 & 1.51 & 0.90 & 245.12 & 91.06 \\
\hline 10 & LY & 4 & M & 1.31 & 3.24 & 6.23 & 2.03 & 0.59 & 30.86 & 1.00 & 397.75 & 105.82 \\
\hline 11 & WMC & 8 & M & 1.04 & 2.35 & 5.07 & 1.00 & 4.09 & 9.71 & BL & 361.15 & 94.65 \\
\hline 12 & DZT & 4 & $\mathrm{M}$ & 0.78 & 2.01 & 2.07 & 3.81 & 0.46 & 14.26 & 0.21 & 395.44 & 136.39 \\
\hline 13 & GYH & 7 & $\mathrm{~F}$ & 0.81 & 4.13 & 5.59 & 2.50 & 0.82 & 9.06 & 11.48 & 282.03 & 141.79 \\
\hline 14 & ZZG & 7 & M & 1.23 & 0.52 & 0.82 & 1.30 & 0.45 & 11.50 & 0.08 & 487.79 & 57.02 \\
\hline 15 & YSH & 4 & $\mathrm{~F}$ & 0.94 & 1.26 & 1.07 & 1.77 & 1.83 & 2.70 & 0.35 & 227.46 & 81.66 \\
\hline 16 & HZX & 4 & $\mathrm{~F}$ & 0.80 & 2.01 & 3.33 & 1.39 & 1.41 & 4.76 & 2.25 & 356.51 & 121.13 \\
\hline 17 & LSC & 7 & M & 0.86 & 0.96 & 2.41 & 2.33 & BL & 1.74 & 1.51 & 171.50 & 131.75 \\
\hline 18 & ZZY & 9 & $\mathrm{~F}$ & 1.34 & 1.07 & 2.67 & 2.88 & 3.47 & 13.91 & 0.05 & 343.05 & 156.07 \\
\hline 19 & HXY & 6 & $\mathrm{~F}$ & 1.12 & 0.94 & 1.42 & 1.77 & 1.18 & 12.39 & BL & 506.50 & 82.34 \\
\hline 20 & WKX & 10 & $\mathrm{~F}$ & 1.14 & 1.89 & 1.67 & 2.56 & 1.51 & 15.83 & 1.32 & 508.67 & 107.17 \\
\hline 21 & RXY & 6 & M & 0.75 & 0.65 & 1.47 & 1.57 & 0.33 & 7.64 & 0.45 & 805.97 & 109.27 \\
\hline 22 & RHY & 6 & M & 1.12 & 2.01 & 1.90 & 1.49 & 3.45 & 9.32 & 1.53 & 843.14 & 132.50 \\
\hline 23 & WHY & 8 & M & 1.06 & 5.28 & 2.04 & 2.03 & 0.78 & 2.72 & 1.38 & 140.73 & 128.21 \\
\hline 24 & LML & 4 & $\mathrm{~F}$ & 0.88 & 1.07 & 3.31 & 3.07 & 2.09 & 13.70 & 0.25 & 110.13 & 65.13 \\
\hline 25 & YDD & 5 & $\mathrm{~F}$ & 0.94 & 3.34 & 4.48 & 2.28 & BL & 4.17 & 1.37 & 83.95 & 54.06 \\
\hline 26 & YZM & 4 & M & 0.46 & 2.03 & 2.15 & 2.46 & BL & 2.19 & 14.66 & 154.83 & 57.70 \\
\hline 27 & LXT & 7 & $\mathrm{~F}$ & 0.59 & 0.86 & 0.71 & 2.89 & BL & 9.59 & 0.62 & 362.68 & 62.15 \\
\hline 28 & CST & 5 & $\mathrm{~F}$ & 1.09 & 3.94 & 2.92 & 1.75 & 0.05 & 7.84 & 1.35 & 244.87 & 95.94 \\
\hline 29 & WQX & 5 & $\mathrm{~F}$ & 1.04 & 3.95 & 2.44 & 1.84 & 3.59 & 2.84 & 0.16 & 289.70 & 80.46 \\
\hline 30 & WJY & 7 & $\mathrm{M}$ & 0.83 & 2.29 & 1.35 & 0.84 & 3.00 & 6.00 & 0.41 & 361.43 & 68.21 \\
\hline 31 & LZM & 6 & $\mathrm{~F}$ & 0.46 & 1.32 & 1.30 & 3.62 & 0.78 & 5.08 & 36.02 & 260.67 & 54.55 \\
\hline 32 & LZY & 7 & M & 1.52 & 1.42 & 1.73 & 3.46 & 0.18 & 3.94 & 0.28 & 124.42 & 78.08 \\
\hline 33 & CYY & 7 & $\mathrm{~F}$ & 0.75 & 2.93 & 2.06 & 8.67 & 3.32 & 11.71 & BL & 220.22 & 118.72 \\
\hline 34 & ZJQ & 7 & $\mathrm{~F}$ & 0.67 & 0.97 & 0.88 & 1.61 & 0.96 & 6.92 & BL & 194.76 & 79.41 \\
\hline
\end{tabular}


Table 2 (Continued)

\begin{tabular}{|c|c|c|c|c|c|c|c|c|c|c|c|c|}
\hline No. & Isocitrate & $\begin{array}{l}\alpha \text {-Keto- } \\
\text { glutarate }\end{array}$ & Succinate & Fumarate & Malate & $\begin{array}{l}\text { Hydroxymethyl- } \\
\text { glutarate }\end{array}$ & $\begin{array}{c}\alpha \text {-Keto- } \\
\text { isovalerate }\end{array}$ & $\begin{array}{c}\alpha \text {-Keto- } \\
\text { isocaproate }\end{array}$ & $\begin{array}{c}\alpha \text {-keto- } \beta \text { - } \\
\text { Methylvalerate }\end{array}$ & $\begin{array}{l}\text { Xanthure- } \\
\text { nate }\end{array}$ & $\begin{array}{l}\beta \text {-Hydroxy- } \\
\text { isovalerate }\end{array}$ & $\begin{array}{l}\text { Methtyl- } \\
\text { malonate }\end{array}$ \\
\hline \multicolumn{13}{|c|}{ Mean $(n=3), \mu \mathrm{g} \mathrm{mg}^{-1}$ creatinine } \\
\hline 1 & 276.89 & 6.08 & 0.93 & BL & BL & 10.86 & 0.17 & 0.05 & 0.65 & 0.59 & 8.03 & 0.20 \\
\hline 2 & 102.61 & 11.15 & 2.37 & BL & BL & 5.44 & 0.12 & 0.05 & 0.57 & 0.47 & 4.73 & 0.41 \\
\hline 3 & 2271.10 & 21.39 & 3.06 & BL & BL & 9.89 & 0.29 & 0.24 & 1.62 & 0.76 & 9.31 & 0.23 \\
\hline 4 & 127.93 & 22.55 & 2.71 & BL & BL & 5.27 & 0.10 & 0.02 & 0.50 & 0.39 & 5.62 & 0.02 \\
\hline 5 & 87.47 & 13.10 & 2.27 & $\mathrm{BL}$ & BL & 4.24 & 0.08 & 0.08 & 0.65 & 0.26 & 9.88 & 0.49 \\
\hline 6 & 60.97 & 14.23 & BL & BL & BL & 3.09 & 0.12 & 0.11 & 0.70 & 0.50 & 11.94 & 0.01 \\
\hline 7 & 0.00 & 2.46 & 0.31 & BL & BL & 4.59 & 0.03 & 0.28 & 0.18 & 0.10 & 4.90 & $\mathrm{BL}$ \\
\hline 8 & 108.20 & 4.41 & 0.77 & 0.67 & BL & 11.01 & 0.32 & 1.86 & 0.61 & 0.22 & 36.47 & 0.50 \\
\hline 9 & 67.74 & 4.73 & 0.28 & $\mathrm{BL}$ & BL & 8.42 & 0.18 & 1.22 & 0.28 & 0.18 & 12.57 & 0.58 \\
\hline 10 & 99.64 & 32.55 & 15.78 & BL & BL & 16.72 & 0.02 & BL & BL & 0.23 & 15.94 & 0.77 \\
\hline 11 & 80.19 & 18.21 & BL & BL & BL & 4.70 & 0.25 & 0.18 & 1.40 & 0.23 & 15.28 & 0.58 \\
\hline 12 & 91.42 & 16.65 & 2.59 & BL & BL & 15.50 & 0.21 & 0.10 & 1.11 & 0.18 & 14.49 & 0.56 \\
\hline 13 & 99.54 & 10.39 & 1.47 & BL & BL & 14.00 & 0.16 & 0.13 & 1.56 & 0.17 & 12.13 & 0.90 \\
\hline 14 & 28.73 & 10.56 & 0.39 & BL & BL & 1.90 & 0.17 & 0.13 & 1.01 & 0.01 & 7.32 & 0.61 \\
\hline 15 & 84.29 & 16.93 & 1.43 & 0.44 & BL & 12.27 & 0.16 & 0.11 & 1.09 & 0.27 & 12.72 & 0.48 \\
\hline 16 & 96.76 & 22.52 & 3.91 & BL & BL & 8.88 & 0.39 & 0.58 & 2.00 & 0.18 & 17.70 & 0.80 \\
\hline 17 & 117.88 & 29.78 & 3.86 & BL & BL & 11.19 & 0.26 & 0.29 & 1.18 & 0.23 & 18.75 & 0.70 \\
\hline 18 & 135.06 & 27.44 & 2.15 & BL & BL & 15.01 & 0.27 & 0.37 & 2.09 & 0.26 & 15.95 & 2.64 \\
\hline 19 & 58.31 & 48.19 & 1.21 & 0.50 & BL & 12.64 & 0.34 & 0.08 & 0.70 & 0.12 & 11.26 & 0.31 \\
\hline 20 & 102.14 & 23.23 & 0.45 & BL & BL & 3.42 & 0.67 & 0.19 & 3.42 & 0.18 & 29.70 & 0.34 \\
\hline 21 & 72.17 & 37.31 & 0.74 & 1.21 & 0.43 & 10.47 & 0.22 & 0.17 & 1.13 & 0.07 & 13.41 & BL \\
\hline 22 & 117.73 & 48.67 & 2.56 & BL & 190 & 15.29 & 0.20 & 0.13 & 1.39 & 0.17 & 15.21 & BL \\
\hline 23 & 106.18 & 43.36 & 0.87 & 0.67 & BL & 15.64 & 0.27 & 0.09 & 1.30 & 0.10 & 12.80 & 0.31 \\
\hline 24 & 68.01 & 14.07 & 3.51 & BL & BL & 12.55 & 0.17 & 0.02 & 0.51 & 0.14 & 4.75 & 0.17 \\
\hline 25 & 50.66 & 7.53 & 1.16 & 0.51 & 3.08 & 10.20 & 0.18 & 0.21 & 0.97 & 0.08 & 10.78 & 0.44 \\
\hline 26 & 37.08 & 7.39 & BL & 0.48 & BL & 8.43 & 0.25 & 0.18 & 1.48 & 0.28 & 14.75 & 0.72 \\
\hline 27 & 48.79 & 25.05 & 0.01 & BL & BL & 9.54 & 0.15 & 0.14 & 0.64 & 0.04 & 6.67 & 0.08 \\
\hline 28 & 91.17 & 17.46 & 4.49 & 0.33 & BL & 19.92 & 0.08 & 0.13 & 0.57 & 0.32 & 9.44 & 0.31 \\
\hline 29 & 77.62 & 35.14 & 11.84 & 0.47 & BL & 16.00 & 0.15 & 0.17 & 0.75 & 0.05 & 5.03 & BL \\
\hline 30 & 54.48 & 26.67 & BL & 0.49 & BL & 13.57 & 0.40 & 0.31 & 2.20 & 0.43 & 22.08 & 1.01 \\
\hline 31 & 44.04 & 17.84 & 1.09 & 0.29 & BL & 11.84 & 0.32 & 0.25 & 1.33 & 0.18 & 21.32 & 0.70 \\
\hline 32 & 86.34 & 13.30 & BL & 0.30 & BL & 9.16 & 0.13 & 0.15 & 0.67 & 0.15 & 13.31 & 0.61 \\
\hline 33 & 69.22 & 28.05 & 0.82 & BL & BL & 13.23 & 0.14 & 0.11 & 0.52 & 0.03 & 9.24 & 0.18 \\
\hline 34 & 179.95 & 20.25 & BL & BL & BL & 10.33 & 0.10 & 0.19 & 0.46 & 0.15 & 11.09 & 0.30 \\
\hline
\end{tabular}

method. The results were expressed as ratios to the urinary creatinine concentration in $\mathrm{mg} / \mathrm{mL}$ creatinine. Each sample was measured three times by MRM for the quantification of each organic acid, and the average value was recorded. Creatinine values were determined by the use of ELISA kits. Coefficient of variations (C.V) of the 20 organic acids ranged from $2.0-$ $12.0 \%$. The average values of $\alpha$-keto- $\beta$-methylvalerate, cisaconitate, isocitrate, hydroxymethylglutarate, $\beta$-hydroxyisovalerate were higher than $95 \%$ reference range. The average values of the rest target organic acids were within the $95 \%$ reference range. Specifically, 31 out of the 34 children had increased level of $\alpha$-keto- $\beta$-methylvalerate; 23 out the 34 children had increased level of hydroxymethylglutarate; 22 out of the 34 children had increased level of cis-aconitate. In detail results are shown in Table 1 . The measured values higher than 95\% reference range were in grey. As we know, vitamins B1, B3 and B5 are necessary for energy pathway of all the cells in the body. As food is broken down, specific compounds such as $\alpha$-ketoglutarate, $\alpha$-ketoisovalerate, $\alpha$-ketoisocaproate, $\alpha$-keto- $\beta$ methylvalerate are formed at steps that requires assistance of $\mathrm{B}$ vitamins. The increased level of $\alpha$-keto- $\beta$-methylvalerate in the present investigation suggests impaired metabolism due to $B$ vitamins insufficiencies or toxic metals. Hydroxymethylglutarate is a metabolic precursor of cholesterol used by the cells to make coenzyme Q10 (CoQ10), which uses oxygen to generate large amounts of energy. An elevation of hydroxymethylglutarate indicates a need for CoQ10 supplementation. cis-Aconitate is involved in the citric acid cycle in the conversion of carbohydrates, fats and proteins into carbon dioxide and water. An increased level of cis-aconitate in urine indicates arginine insufficiency for ammonia clearance through the urea cycle or problems with protein metabolism among the investigated children. According to the nutritional and healthy survey of Chinese residents in 2019, the average food consumption containing B vitamins is $80 \%$ lower than the suggested amount, which means B vitamins deficiencies are normal among Chinese population. In this study, a high level of marker organic acids also proved that $\mathrm{B}$ vitamins insufficiencies were not exceptional among the investigated children. A low consumption of $\mathrm{B}$ vitamins causes impaired metabolism in the cell, resulting in high urinary organic acids accumulation. Due to a small sample size, no clinical decision can be concluded from this study. More samples are currently being evaluated for a clinical validation of the investigated organic acids. The preliminary data in this study confirmed the suitability of the method for the rapid and accurate quantification of the target organic acids in urine. 


\section{Conclusions}

A rapid quantification of 20 urinary organic acids by HPLC mass spectrometry was developed and applied to study nutritional individuality in Chinese children. The method was proved to be robust and reproducible in analyzing urine samples. A preliminary statistical analysis of 34 samples revealed the potential diagnostic power of the target organic acids. This assay involved sample pretreatments to exclude urinary interference and accurate quantification of the target organic acids without derivatization. The findings from this study indicated that the abnormalities in urinary organic acids in a Chinese children population, which reveal evidence of functional inadequacy of specific nutrients in children. This method with high sensitivity and accuracy greatly reduced the analysis time, and is especially suitable for large-scale sample analysis. Next, we are about to apply this assay in an epidemiology study to investigate nutrient insufficiencies among Chinese children population.

\section{Acknowledgements}

This study was supported by Public Health and Preventive Medicine Provincial Experiment Teaching Center at Sichuan University and Food Safety Monitoring and Risk Assessment Key Laboratory of Sichuan Province and National nature science foundation of China 81600603 . This study was also supported by Sichuan Science and Technology Program (No. 2020JDRC0164).

\section{References}

1. S. R. Pieczenik and J. Neustadt, Exp. Mol. Pathol., 2007, $83,84$.

2. J. Pizzorno and M. Murray, "Textbook of Natural Medicine $4 e$ ", 2012, Elsevier-Health Sciences Division, Churchill Livingstone Inc, 28.

3. D. P. Simpson, Am. J. Physiol., 1983, 244.

4. S. E. Bentler, A. J. Hartz, and E. M. Kuhn, J. Clin. Psychiatry, 2005, 66, 625.
5. J. H. Park, K. J. Niermann, and N. Olsen, Curr. Rheumatol. Rep., 2000, 2, 131.

6. R. F. Butterworth, J. Inherit. Metab. Dis., 1998, 21, 6.

7. C. Burdette, "How to Assess Patient Biochemical and Nutritional Individuality through Organic Acid Testing", Townsend Letter for Doctors and Patients, Issue 270, The Townsend Letter Group.

8. A. Kumps, P. Duez, and Y. Mardens, Clin. Chem., 2002, 48, 708.

9. K. Shibata and M. Sakamoto, J. Nutr. Sci. Vitaminol., 2016, 62, 220.

10. A. Klupczynska, S. Plewa, N. Sytek, W. Sawicki, P. Dereziński, J. Matysiak, and Z. J. Kokot, J. Pharm. Biomed. Anal., 2018, 159, 229.

11. H. Awad, K. J. H. Allen, D. J. Adamko, and A. El-Aneed, J. Chromatogr. B, 2019, 1122-1123, 29.

12. W. J. Visek, Cancer Res., 1992, 52(Supplement), 2082s.

13. R. Baziramakenga, R. R. Simard, and G. D. Leroux, Soil Biol. Biochem., 1995, 27, 349.

14. C. W. Klampfl, W. Buchberger, and P. R. Haddad, $J$. Chromatogr. A, 2000, 881, 357.

15. F. Chinnici, U. Spinabelli, C. Riponi, and A. Amati, J. Food Compos. Anal., 2005, 18, 121.

16. T. Acunha, C. Ibáñez, V. García-Cañas, C. Simó, and A. Cifuentes, Electrophoresis, 2016, 37, 111.

17. J. Regueiro, A. Vallverdú-Queralt, J. Simal-Gándara, R. Estruch, and R. Lamuela-Raventós, J. Agric. Food Chem., 2013, 61, 6763.

18. J. Kałuzna-Czaplinska, Clin. Biochem., 2011, 44, 686.

19. B. J. Verhaeghe, M. F. Lefevere, and A. P. De Leenheer, Clin. Chem., 1988, 34, 1077.

20. A. Liu, M. M. Kushnir, W. L. Roberts, and M. Pasquali, J. Chromatogr. B, 2004, 806, 283.

21. G. Wittmann, E. Karg, A. Mühl, O. A. Bodamer, and S. Türi, J. Inherit. Metab. Dis., 2008, 31, 73.

22. C. Kumari, B. Varughese, S. Ramji, and S. Kapoor, Indian J. Clin. Biochem., 2016, 31, 414.

23. J. Wang and W. C. Schnute, Rapid Commun. Mass Spectrom., 2009, 23, 3439.

24. B. Tan, Z. Lu, S. Dong, G. Zhao, and M. S. Kuo, Anal. Biochem., 2014, 465, 134.

25. J. Fiori, E. Amadesi, F. Fanelli, C. V. Tropeano, M. Rugolo, and R. Gotti, J. Pharm. Biomed. Anal., 2018, 150, 33. 